(c) 2018 IEEE. Personal use of this material is permitted. Permission from IEEE must be obtained for all other uses, in any current or future media, including reprinting/republishing this material for advertising or promotional purposes, creating new collective works, for resale or redistribution to servers or lists, or reuse of any copyrighted component of this work in other works. 


\title{
A Monte Carlo simulation study of scatter fraction and the impact of patient BMI on scatter in long axial field-of-view PET scanners
}

\author{
Amir Ghabrial $^{\mathrm{a}, \mathrm{b}}$, Daniel R. Franklin ${ }^{\mathrm{b}}$, Habib Zaidi ${ }^{\mathrm{a}, \mathrm{c}, \mathrm{d}}$ \\ ${ }^{a}$ Division of Nuclear Medicine and Molecular Imaging, Geneva University Hospital, \\ CH-1211 Geneva, Switzerland \\ ${ }^{b}$ University of Technology Sydney, Ultimo NSW 2007, Australia \\ ${ }^{c}$ Department of Nuclear Medicine and Molecular Imaging, University of Groningen, \\ University Medical Center Groningen, 9700 RB Groningen, Netherlands \\ ${ }^{d}$ Department of Nuclear Medicine, University of Southern Denmark, DK-500, Odense, \\ Denmark
}

\begin{abstract}
The NEMA NU-2 standard describes a protocol for measurement of scatter fraction (SF) using an axially-aligned line source, offset at $45 \mathrm{~mm}$ from the central axis, in a cylindrical polyethylene phantom. In this work, which is an extension of our preliminary results previuosly published in the Proceedings of IEEE NSS/MIC 2018 [1], we aim to evaluate the performance of the the NEMA NU-2 $\mathrm{SF}$ protocol in a Siemens Biograph mCT PET/CT whole-body scanner and a long axial field-of-view (LAFOV) total-body PET scanner to determine whether modifications to the NEMA NU-2 SF protocol are needed for the characterisation of scatter in such scanners. In addition, we evaluate the impact of patient body mass index (BMI) on SF in a LAFOV scanner. The Siemens Biograph $\mathrm{mCT}$ and a typical LAFOV PET scanner were modelled in GATE. Monte Carlo simulations were performed to validate the $\mathrm{mCT}$ scanner model against published experimental results. SF was estimated using a modified NEMA NU-2 protocol with variable radial offsets on both scanners and compared to ground truth SF measurements obtained with a uniform-activity cylindrical phantom. Correlation between BMI and SF in the LAFOV scanner was evaluated by simulating anthropomorphic phantoms with different BMIs and realistic ${ }^{18} \mathrm{~F}-\mathrm{FDG}$ distributions, together with uniformly-filled $200 \mathrm{~cm}$ long cylindrical phantoms with equivalent effective diameters. The optimal offset was found to be either $60 \mathrm{~mm}$ or $80 \mathrm{~mm}$, depending on the chosen optimality metric. We conclude that modifications to NEMA NU-2 are required for accurate SF characterisation in whole-body and LAFOV scanners. Finally, SF in anthropomorphic phantoms with realistic tissue concentrations of ${ }^{18} \mathrm{~F}-\mathrm{FDG}$ was found to be strongly cor-
\end{abstract}

\footnotetext{
${ }^{\star}$ Corresponding Author: Daniel R. Franklin, School of Electrical and Data Engineering, University of Technology Sydney, Australia Tel: +61 295142495.

Email address: Daniel.Franklin@uts.edu.au (Daniel R. Franklin)
} 
related with $\mathrm{SF}$ in an equivalent-volume cylindrical phantom for the LAFOV PET scanner; BMI was also found to strongly positively correlate with the SF.

Keywords: Biograph mCT, GATE, EXPLORER, scatter fraction, long axial field of view, NEMA, anthropomorphic phantom 


\section{Introduction}

A significant recent trend in PET has been the growing interest in total-body PET, in which the axial field-of-view is extended to cover the full length of an adult human (typically around two metres) [2]. Such long axial field-of-view (LAFOV) scanners offer numerous benefits compared to classical short axial FOV scanners, including (i) higher sensitivity leading to improved image quality for a given radiotracer dose, or, equivalently, a reduced image acquisition time for a given injected dose, reducing patient motion artefacts (ii) the ability to scan an entire adult simultaneously in a single bed position; (iii) the ability to perform dynamic (4D) whole-body studies for pharmacokinetic modeling over a longer period of time; and (iv) the ability to perform ultra-low dose imaging studies for example, allowing PET to be used with pediatric patients and for multiple consecutive follow-up scans for patient monitoring to evaluate the response to treatment [3]. The high sensitivity and other benefits come at a considerably increased cost, implementation complexity and image reconstruction burden (both in terms of memory and computational complexity).

LAFOV PET offers significantly greater overall sensitivity and count-rate performance compared to classical PET scanners. However, certain aspects of scanner performance are degraded due to the extension of the axial field of view (AFOV). In particular, the rates of random and scattered coincidences and the system dead-time tend to increase, with the scatter fraction asymptotically approaching a limit that depends on the scanner diameter and other geometric factors [4]. A number of LAFOV PET prototypes that are longer than current commercial PET scanners have been proposed and are being constructed. Among the first LAFOV PET scanners was a prototype developed by a Japanese group with $68.5 \mathrm{~cm}$ axial length using BGO scintillation crystals [5]. Another prototype with an axial length of $53 \mathrm{~cm}$ using LSO panel detector modules was developed by Siemens Healthcare [6]. An alternative approach pioneered by Crespo et al. utilized resistive plate chamber (RPC) technology for a costeffective approach to photon detection in a high resolution depth-of-interaction LAFOV PET system [7].

The first true total-body PET scanner, uEXPLORER, was developed by researchers at the University of California Davis in collaboration with United Imaging Healthcare, and aimed to provide a $200 \mathrm{~cm}$ AFOV. The first simulation model of EXPLORER was loosely based on Siemens Biograph mCT PET detector modules [8]. The same group also designed and built a mini-EXPLORER prototype for non-human primate studies with a $45.7 \mathrm{~cm}$ AFOV[9], while the a second-generation small-scale clinical prototype, MiniEXPLORER II, was developed for human brain and companion animal studies, and incorporated a CT scanner with a pair of 16-detector PET sections, for a $48.3 \mathrm{~cm}$ total AFOV[10]. The final iteration of the EXPLORER design was a fully integrated $194 \mathrm{~cm}$ long scanner $[2,3]$. The first quantitative image reconstruction method for this scanner was developed by Zhang et al. and demonstrated using a Monte Carlo simulation of a realistic activity distribution in an anthropomorphic phantom; estimates of the counts of true and scattered photons together with scatter frac- 
Table 1: Scatter fractions of several commercial PET and PET/CT scanners reported in the literature.

\begin{tabular}{|c|c|c|}
\hline Scanner & Scatter Fraction & $\overline{\text { Reference }}$ \\
\hline GE Discovery-690 / Discovery MI PET/CT & $37 \%$ & {$[14,15]$} \\
\hline Philips Gemini TF PET/CT Big Bore & $31 \%$ & [16] \\
\hline Philips Gemini TF PET/CT 64 & $30 \%$ & {$[17]$} \\
\hline Philips Vereos digital scanner & $33.9 \%$ & [18] \\
\hline Canon Celesteion large-bore PET/CT & $37.3 \%$ & [19] \\
\hline Early Siemens Biograph ${ }^{\mathrm{TM}} 6 \mathrm{PET} / \mathrm{CT}$ & $33.4 \%$ & {$[20]$} \\
\hline Siemens Biograph TruePoint TrueV & $32.7 \%$ & [21] \\
\hline Siemens Biograph mCT & $33.2 \%$ & [21] \\
\hline Siemens Biograph Vision & $38.7 \%$ & {$[22]$} \\
\hline United Imaging uEXPLORER & $36.3 \%$ & {$[23]$} \\
\hline
\end{tabular}

tion as a function of ring difference were calculated and the relative contributions of single and multiple scatter events are reported [11].

A collaboration between Ghent University and Vrije Universiteit Brussels is developing a one-metre AFOV high-resolution total-body PET scanner (dubbed PET20.0), which will offer higher spatial resolution than EXPLORER and is intended for paediatric patients [12]. Similarly, a group at the University of Pennsylvania is building a silicon photomultiplier-based $70 \mathrm{~cm}$ AFOV time-offlight (TOF) PET/CT scanner to enable whole-body dynamic imaging with high sensitivity, with a modular architecture that could be extended to up to $140 \mathrm{~cm}$ via the addition of more PET rings [13].

The scatter fraction (SF) of a PET scanner is an important performance metric, which quantifies the proportion of total detected photons which have undergone scatter prior to detection. It is a function of several physical and geometric parameters, such as the scintillation detector energy resolution and window, detector geometry and solid angle coverage; it also depends on the size and composition of the object being imaged. An intrinsic trade-off exists between scatter fraction and sensitivity - the energy window can be narrowed to achieve a lower scatter fraction, but sensitivity will also be reduced. The SF is defined as the fraction of all detected coincidence events in which at least one of the two emitted annihilation photons are scattered prior to detection, under the condition that the number of random coincidences events is less than $1 \%$ of the rate of trues. The rate of randoms can be estimated by evaluating the coincidence rate obtained when one channel is delayed. Scatter fractions for several commercial PET scanners are listed in Table 1.

Scattered events cause misplacement of lines of response, since one or both of the annihilation photons deviates from the original trajectory while traversing the imaging subject or other matter prior to detection. It is therefore desirable for the scatter fraction to be as low as possible while maintaining satisfactory scanner sensitivity; this will reduce artefacts and bias in the reconstructed PET image and maximise the quantitative integrity of the acquired coincidence 
events. The majority of scatter occurs within the patient rather than the detectors (as these are compact and dense in modern PET systems) [24].

Several alternative approaches are available for correcting the effect of scatter in PET imaging. The scatter fraction can be reduced by either narrowing the energy acceptance window or using coarse septa or other shielding devices to limit scatter from outside of the field of view; however, the former approach reduces sensitivity, while the latter is not appropriate for total-body scanners [25]. If list-mode data is available (including the energy of each coincident photon), spectral techniques can be used to estimate the contribution of scattered events to the image by discriminating between the expected spectra of unscattered vs. scattered photons [25]. Scatter correction based on Monte Carlo simulation of photon propagation together with emission and transmission images are very accurate, but computationally complex; they offer the advantage of being able to model multiple photon scatter events $[25,26,11]$. More recently, many researchers have investigated scatter analysis/synthesis methods based on deep learning, which can be considerably less computationally intensive while accounting for multiple scattering events [27]. PET systems with time-of-flight acquisition capabilities such as the popular Siemens Biograph mCT system offer additional opportunities for scatter correction due to their ability to more precisely localise the origin of scatter [28, 29].

Monte Carlo simulations are widely used for evaluating and optimising the performance of nuclear medical imaging modalities such as CT, SPECT and PET [30]. They are particularly relevant for the development and evaluation of image reconstruction algorithms and correction schemes which aim to improve image quality and quantitative accuracy. Monte Carlo simulations have been widely utilised for the development of scatter compensation methods [31, 32], to validate practical scatter correction methods [33, 34], and to develop new scatter corrections algorithms [35, 36]. Likewise, the performance of LAFOV PET scanners have been evaluated using Monte Carlo simulation tools [8, 37, 4, 38]. Evaluation of scatter fraction in these simulations is normally performed using the NEMA NU-2 protocol. However, it has been suggested previously that the NEMA NU-2 protocol, which specifies a $70 \mathrm{~cm}$ long cylindrical polyethylene phantom for scatter fraction estimation scans with a radially-offset uniform axial line source, is inappropriate for accurate estimation of the scatter fraction that will be observed in a PET scan of an anthropomorphic object with a realistic activity distribution [39].

In this work, which is an extension of our preliminary results previously published in the Proceedings of IEEE NSS/MIC 2018 [1], SFs for a LAFOV PET scanner are evaluated using Monte Carlo simulations implemented in the Geant4 Application for Tomographic Emission (GATE) [40], and compared to that of a Siemens Biograph mCT PET scanner using a variety of scattering phantoms based on variants of those defined in the National Electrical Manufacturers Association (NEMA) NU-2 2007 protocol [41]. Several modifications to the existing NEMA NU2-2007 standard, aiming at improving the accuracy of the estimated SF, are proposed and evaluated for both the $\mathrm{mCT}$ and LAFOV scanner designs. Additionally, we investigate the relationship between patient 
body mass index (BMI) and scatter fraction using a set of anthropomorphic phantoms - a clinically relevant consideration due to the increasing proportion of patients who are overweight or obese, which is expected to result in elevated scatter fractions.

Section 2 describes the methodology used in this paper. Section 3 presents the key findings, which are discussed in depth in Section 4 . Section 5 summarises the overall findings of this research and suggests several avenues for future work.

\section{Materials and methods}

This work comprises two separate contributions:

- An investigation of modifications to the NEMA NU-2 SF evaluation protocol to improve its suitability for use with LAFOV scanners. The methods used to evaluate SF and determining the optimal radial offset are adapted from Ferrero et al. and are discussed in detail in Section 2.2 [42].

- An evaluation of the relationship between BMI and SF in anthropomorphic phantoms, described in detail in Section 2.4.

The reference scanner geometries and simulation parameters used throughout the paper are described in Section 2.1.

\subsection{Scanner geometry and simulation parameters}

The design parameters for the $\mathrm{mCT}$ and LAFOV PET scanners are summarised in Table 2. The mCT model is based on the commercially-available scanner [21, 43], while the LAFOV scanner geometry is based on the model proposed by Zhang et al [11]. The $0.08 \mathrm{~mm}$ gap between adjacent crystals in the LAFOV scanner represents a powder-based inter-crystal reflective coating. The LAFOV scanner system model simulated in GATE is shown in Figure 2.1.

Version 7.0 of the GATE Monte Carlo simulator, built with Geant4 version 9.6.p03, was used to perform all simulations of the Siemens Biograph mCT and LAFOV scanners in this work [40]. Models of the cylindrical and anthropomorphic phantoms were also developed for use with both scanners. The Biograph mCT PET scanner simulation model was previously validated through comparison with experimental measurements of spatial resolution, sensitivity, SF and NECR using the NEMA NU2-2007 standard [44]. All possible lines of response are included.

\subsection{NEMA-like scatter fraction protocol}

The NEMA NU 2-2007 standards are used to evaluate and validate a GATE model of the Siemens Biograph mCT PET/CT system [41]. Although newer versions of these standards (NEMA NU 2-2012 [45] and NU 2-2018 [46]) have been published, the benchmark data used for validation are based on the 2007 standard [21]; therefore, although the 2007 standard is used throughout this work, the results still apply to the 2012 and 2018 revisions. 
Table 2: Parameters for Siemens Biograph mCT and LAFOV PET scanners as used in all GATE simulations

\begin{tabular}{lll}
\hline Parameter & mCT & LAFOV \\
\hline Number of block rings & 4 & 36 \\
Detector blocks per ring & 48 & 48 \\
Scintillator material & LSO & LSO \\
Crystals per block & $13 \times 13=169$ & $15 \times 15=225$ \\
Crystals per axial ring & 624 & 720 \\
Axial FOV (mm) & 218 & 1966 \\
Transaxial FOV & 700 & 700 \\
Coincidence time window (ns) & 4.1 & 5.5 \\
Energy window (keV) & $435-650$ & $435-650$ \\
Energy resolution (\%) & 11.7 & 13 \\
Adjacent block rings gap (mm) & 4.0 & 3.42 \\
Crystal size (mm) & $4 \times 4$ & $3.34 \times 3.34$ \\
Crystal length (thickness) (mm) & 20 & 20 \\
Detector ring diameter (mm) & 842 & 800 \\
Time of Flight resolution (ps) & 527.5 & 530 \\
Number of crystals in one module & $13 \times 13=169$ & $15 \times 15=225$ \\
Number of crystals in one sector & $169 \times 4=676$ & $225 \times 36=8100$ \\
Number of crystals & $676 \times 48=32448$ & $8100 \times 36=388800$ \\
\hline
\end{tabular}

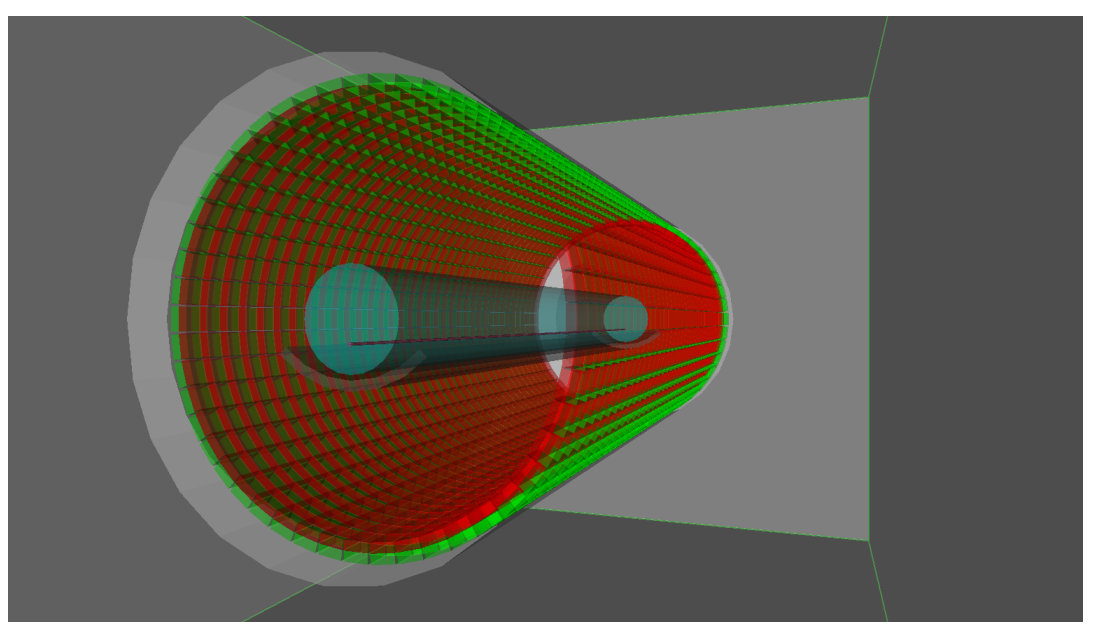

Figure 1: Graphical representation of the GATE model of the LAFOV PET scanner with NEMA-like cylindrical phantom (20 cm diameter, $200 \mathrm{~cm}$ length), centred on the scanner's axial FOV, with the line source inserted at a radial displacement of $45 \mathrm{~mm}$ from the central axis. The cylinder is placed on the bed of the scanner. 
The NEMA NU 2-2007 protocol uses a $70 \mathrm{~cm}$ cylindrical polyethylene phantom with an axially-oriented line source positioned at a fixed radial offset of $45 \mathrm{~mm}$. There is some evidence that using this source and phantom combination in longer total-body scanners results in an underestimation of actual scatter fraction compared to a realistic clinical scan, due to increased scatter from the axial extremities of the patient (or phantom) [23, 47].

In this work, the phantom (described in Section 2.3.1) is extended to fill the full axial FOV of the LAFOV scanner, while the radial offset of the line source is varied over a range from 0 to $95 \mathrm{~mm}$. The line source activity concentration is fixed at $45 \mathrm{kBq} / \mathrm{mL}$. For comparison, a uniform cylindrical phantom with a length of 2 metres and a diameter of $20 \mathrm{~cm}$ was simulated and the ground truth scatter fraction measured by direct scoring of the scattered and unscattered photons. Photons which scatter in the phantom and bed are both counted as scattered events in this study; scatter in the detector crystals is not.

The sinograms for the collected data are single-slice rebinned (SSRB) and stored as parallel sinograms using STIR [48], where each profile has a diameter of $240 \mathrm{~mm}$. This is $40 \mathrm{~mm}$ wider than the scatter phantom, since the FOV depends on the specific scanner geometry. In each sinogram, the transverse FOV is restricted over the extent of the phantom by setting all bins outside of a radial distance of $120 \mathrm{~mm}$ to zero. Then, each projection in the sinogram is shifted so that radial bins with the maximum value amongst all projections are aligned at the centre of the sinogram. A sum of these shifted projections is generated, with a count density distribution at the centre of the sinogram around the peak maximum counts. It is assumed that all true coincidences (plus a background of scatter and randoms) are located within $\pm 20 \mathrm{~mm}$ of the peak value, and that there are no true events (only scatter and randoms) beyond the $40 \mathrm{~mm}$ strip around the amplitude peak of the sinogram.

The scattered events $C_{s}$ under the peak are estimated by calculating the pixel intensity at the left and right edges of the $40 \mathrm{~mm}$ strip. Then, the average of the pixel counts is computed and the result (including fractional values) multiplied by the pixel number inside the $4 \mathrm{~cm}$ strip and adding the result to the pixel counts outside the strip. The result provides an estimate of the total number of scatter events $C_{s}$. The total event count $C_{t o t}$ is computed as the summation of all the pixel values for all the projections. The true count, $C_{\text {true }}$, is calculated using (1):

$$
C_{\text {True }}=C_{\text {tot }}-C_{s}
$$

Total SF is then estimated from the weighted average of the SF values of all slices using (2) [41]:

$$
S F=\frac{C_{s}}{\left(C_{\text {True }}+C_{s}\right)}
$$

The SFs were evaluated using a NEMA scatter phantom and estimated using direct binning from the available scatter and prompt coincidence counts, using (2). 
Ground truth scatter fraction is obtained directly from the GATE record of prompt events, which record the number and type of scatter events (and where the scatter occurred) prior to detection each half of the coincidence pair.

\subsection{Phantom models}

\subsubsection{NEMA scatter phantom}

The NEMA NU-2 performance tests are the standard benchmark used for quantitative performance evaluation and comparison of clinical PET scanners. The standard sequence of phantom-based measurements evaluates each of the key PET scanner performance metrics, including spatial resolution, SF, noise equivalent count rates, sensitivity, image contrast and accuracy of the image correction methods.

SF is evaluated using a solid polyethylene cylinder $\left(\rho=0.96 \mathrm{~g} / \mathrm{cm}^{3}\right)$ with an external diameter of $200 \mathrm{~mm}$ with a length of $700 \mathrm{~mm}$. An off-axis cylindrical hole, $6.4 \mathrm{~mm}$ in diameter, is located at a single fixed radial distance of $45 \mathrm{~mm}$, parallel to the central axis of the phantom cylinder [41]. The line source is an $800 \mathrm{~mm}$ long hollow polyethylene tube with an inside diameter of $3.2 \mathrm{~mm}$ and an outside diameter of $4.8 \mathrm{~mm}$, which is filled with ${ }^{18} \mathrm{~F}$ solution with activity concentrations of up to $45 \mathrm{kBq} / \mathrm{ml}$ and inserted into the hole in the phantom.

In this work, the length of the phantom and line source are extended to $200 \mathrm{~cm}$ in order to cover the entire field of view of the LAFOV scanner, since the principal application of such scanners will be to image the entire body simultaneously (with activity - and scatter - distributed throughout the entire body). Additionally, the radial offset for the axially-oriented line source is varied over a range of 0 to $95 \mathrm{~mm}$ to determine the offset that will provide the best match to the scatter fraction obtained from a uniform flood-filled cylinder phantom.

\subsection{BMI and SF in anthropomorphic phantoms}

An anthropomorphic phantom model based on the standard ICRP human phantom was used to produce a set of realistic voxelised human 3D phantoms covering a range of body mass index (BMI) values (Figure 2) [49]. These models were then used as input to Monte Carlo simulations for SF estimations on the LAFOV PET scanner. Unlike the cylindrical NEMA phantoms, the anthropomorphic computational phantoms comprise organs and anatomical structures and have complex activity distributions based on realistic uptake of ${ }^{18} \mathrm{~F}-\mathrm{FDG}$ in the body, based on an injected dose of $3.5 \mathrm{MBq} / \mathrm{kg}$ [49] and pharmacokinetic parameters for different organs as listed in ICRP Publication 106 [50]. The phantom includes fourteen different functional organs and anatomical structures, including the bladder, soft tissues/muscle, blood pool, brain, heart myocardium, urethra, liver, lungs, spine bone, spleen, ribs, stomach and kidney [51]. The simulated anthropomorphic phantoms are rendered with a voxel size of $1.775 \times 1.775 \times 4.84 \mathrm{~mm}^{3}$. The phantoms' dimensions (in voxels and $\mathrm{cm}$ ) and BMIs are listed in Table 3 . 

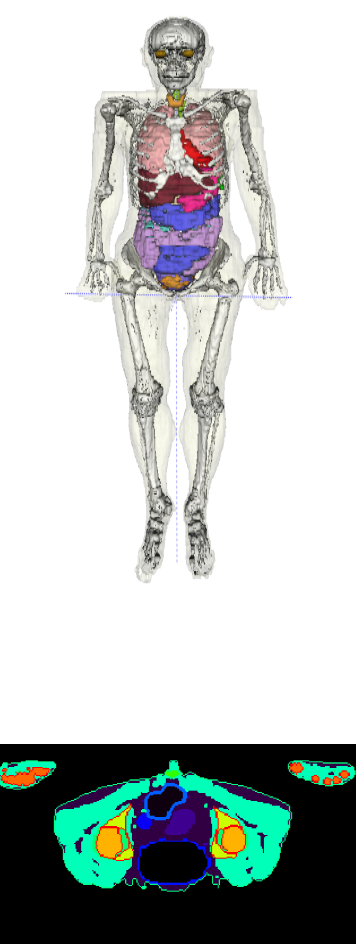

(a) $\mathrm{BMI}=23.4$
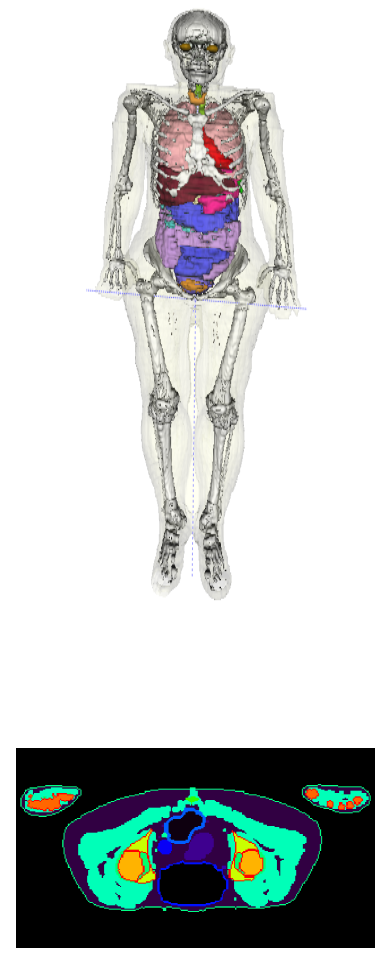

(b) $\mathrm{BMI}=26.0$
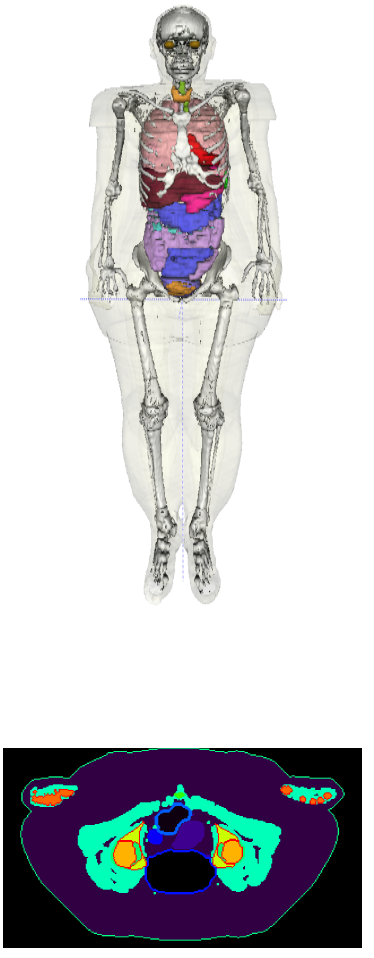

(c) $\mathrm{BMI}=39.5$

Figure 2: Illustration of three of the twelve voxelised anthropomorphic computational phantoms (together with transverse sections through the bladder). The phantoms are based on realistic human models with different BMIs using the ICRP model as an anchor phantom. The phantoms are shown in the order of increasing effective diameter as per Table 3. 
Table 3: Geometry of computational anthropomorphic phantoms and the equivalent cylindrical phantoms.

\begin{tabular}{lccll}
\hline $\begin{array}{l}\text { Phantom } \\
\text { number }\end{array}$ & $\begin{array}{l}\text { BMI } \\
\left(\mathrm{kg} / \mathrm{m}^{2} \text { dimensions }\right. \\
\text { (voxels) }\end{array}$ & $\begin{array}{l}\text { Phantom } \\
\text { length } \\
(\mathrm{m})\end{array}$ & $\begin{array}{l}\text { Effective } \\
\text { Diameter } \\
(\mathrm{mm})\end{array}$ \\
\hline (a) & 21.8 & $355 \times 158 \times 338$ & 1.64 & 208.5 \\
(b) & 23.8 & $345 \times 154 \times 329$ & 1.59 & 209.6 \\
(c) & 22.6 & $299 \times 137 \times 348$ & 1.68 & 211.1 \\
(d) & 23.4 & $349 \times 155 \times 339$ & 1.64 & 212.4 \\
(e) & 21.2 & $369 \times 165 \times 366$ & 1.77 & 213.6 \\
(f) & 26.0 & $335 \times 151 \times 348$ & 1.68 & 222.3 \\
(g) & 29.4 & $330 \times 150 \times 339$ & 1.64 & 224.7 \\
(h) & 31.7 & $301 \times 150 \times 349$ & 1.69 & 226.3 \\
(i) & 35.3 & $301 \times 160 \times 330$ & 1.60 & 228.2 \\
(j) & 39.5 & $301 \times 171 \times 337$ & 1.63 & 232.5 \\
(k) & 37.3 & $301 \times 168 \times 359$ & 1.74 & 234.0 \\
(l) & 36.2 & $301 \times 167 \times 367$ & 1.78 & 234.6 \\
\hline
\end{tabular}

\subsubsection{Cylindrical phantoms}

Twelve cylindrical phantoms were also simulated, each with a length of $200 \mathrm{~cm}$, and a diameter equivalent to the mean effective diameter (ED) of each of the twelve anthropomorphic phantoms. This diameter was determined using the method described in the AAPM report 204 [52], by calculating the average of the anterior-posterior $(A P)$ dimension and the lateral $(L A T)$ dimension for each of the twelve anthropomorphic phantoms using (3) for each axial slice of the phantom, and then computing an overall mean ED. Thus, the average cross-sectional area of the anthropomorphic phantom is represented by a circle with a diameter equal to the mean ED.

$$
E D=\sqrt[2]{L A T \times A P}
$$

\section{Results}

Figure 3 shows the spectral energy distribution obtained by Monte Carlo simulation of a water-filled cylindrical phantom and a positron-emitting ${ }^{18} \mathrm{~F}$ source. The scattered events in the energy pulse-height distribution have been separated according to the order of scattering.

The SF for the mCT PET scanner calculated using Monte Carlo simulations performed with the standard NEMA phantom and using an energy window of $435-650 \mathrm{keV}$ is $34.35 \%$. This result is consistent with NEMA results obtained in an experimental study using a source activity of $1 \mathrm{kBq} / \mathrm{ml}$, confirming the validity of the GATE simulation model [21].

The modified NEMA simulations (extending the phantom to $200 \mathrm{~cm}$ with a source activity of $1 \mathrm{kBq} / \mathrm{ml}$ with a radial offset of $45 \mathrm{~mm}$ for the LAFOV 


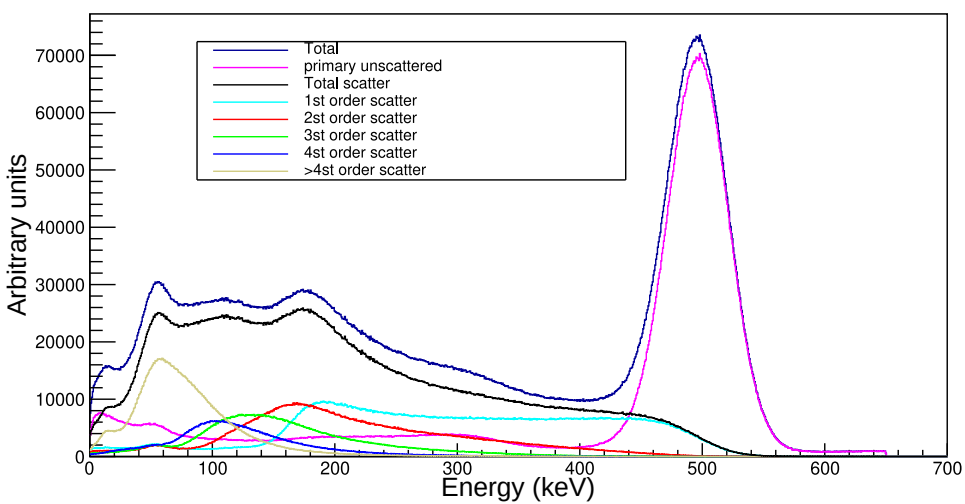

(a) $\mathrm{mCT}$

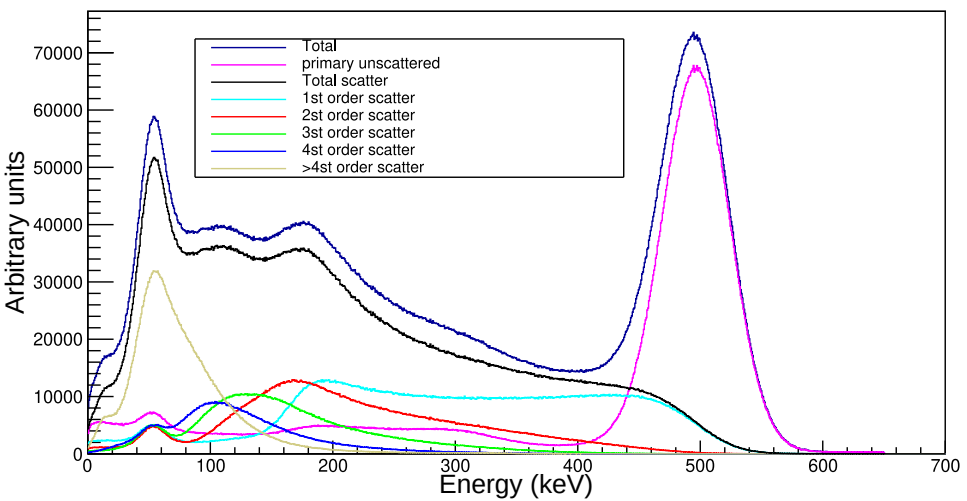

(b) LAFOV

Figure 3: Plots of the energy spectra calculated by the Monte Carlo method in the mCT and LAFOV PET scanners, showing the contributions to each from unscattered and scattered photons. The simulated phantom is a cylinder with a diameter of $20 \mathrm{~cm}$ and lengths of $70 \mathrm{~cm}$ and $200 \mathrm{~cm}$ for the $\mathrm{mCT}$ and LAFOV scanners, respectively, uniformly filled with an ${ }^{18} \mathrm{~F}$ solution. The spectrum of unscattered photons is shown in magenta, while the spectra due to scattered photons (solid black line) is separated into the contributions from different orders of photon scattering (shown in a range of colours). The distributions of scattered and unscattered photons are added to give the total spectra (solid blue line). The mCT and LAFOV scanner energy resolutions are $11.7 \%$ and $13 \%$, respectively. 


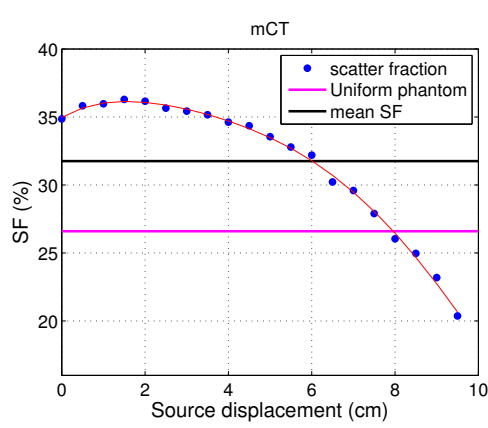

(a) $\mathrm{mCT}$

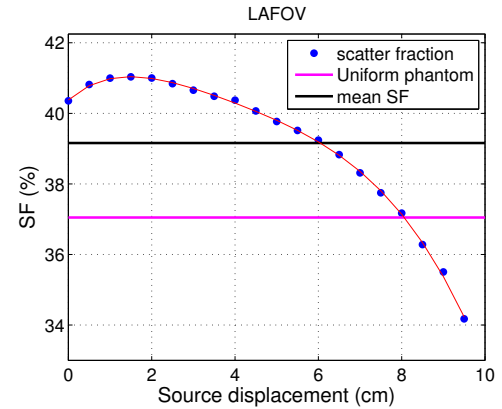

(b) LAFOV

Figure 4: Plots of scatter fraction as a function of the source displacement, obtained using NEMA/NEMA-like polyethylene phantoms with a diameter of $20 \mathrm{~cm}$ and lengths of $70 \mathrm{~cm}$ and $200 \mathrm{~cm}$ for the mCT and LAFOV PET scanners, respectively (C)IEEE; reproduced with permission [1])

PET scanner result in a SF estimate of $40 \%$. However, the simulation of a uniformly-filled cylindrical phantom result in a lower scatter fraction of $37.1 \%$. This indicates that the results obtained with the NEMA/NEMA-like phantom with a $45 \mathrm{~mm}$ offset significantly overestimate the SF value that would occur during a clinical scan. The SF curve intersects the uniform phantom value at a radial displacement of approximately $80 \mathrm{~mm}$, which is the same displacement as that previously reported as being optimal (also in terms of agreement with the uniform phantom) for the GE Discovery STE scanner [42]. On the other hand, the SF curve intersects with the mean SF value obtained from all simulations at different radial displacements at about $60 \mathrm{~mm}$ radial displacement from the phantom centre, for both the $\mathrm{mCT}$ and the LAFOV PET scanners, as illustrated in Figure 4(a) and 4(b), respectively.

\subsection{BMI and SF in anthropomorphic phantoms}

The SFs obtained for uniformly-filled NEMA-like phantoms with diameters ranging between $100 \mathrm{~mm}$ and $600 \mathrm{~mm}$ (in steps of $50 \mathrm{~mm}$ ) and lengths of $70 \mathrm{~cm}$ and $200 \mathrm{~cm}$ for the simulated Biograph $\mathrm{mCT}$ and LAFOV PET scanners, respectively, are shown in Figure 5 together with a fitted second-order polynomial.

The correlation of between the anthropomorphic phantom body mass index (BMI) and the SF is 0.924, as shown in Figure 6. As the effective diameter of the anthropomorphic phantoms increase, the SF of both the anthropomorphic phantoms and the equivalent cylindrical phantoms increase (Figure 7(a)-7(b)). The correlation between SFs for different anthropomorphic phantoms and the equivalent cylindrical phantom SF is 0.98 (Figure 7(c)).

Scatter fractions for the anthropomorphic phantoms and the equivalent cylindrical phantoms simulated using the LAFOV PET scanner are listed in Table 4. Simulations were repeated 11 times, with the standard deviation equal to $0.23 \%$. 


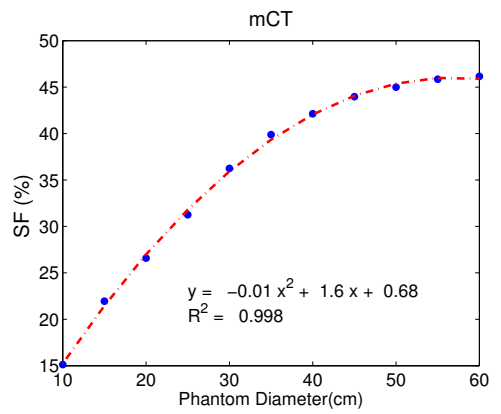

(a) $\mathrm{mCT}$

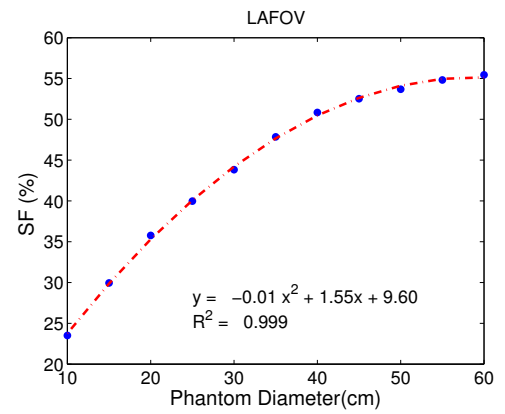

(b) LAFOV

Figure 5: Scatter fraction vs. diameter of uniformly filled NEMA/NEMA-like phantoms (70 $\mathrm{cm}$ and $200 \mathrm{~cm}$ for the $\mathrm{mCT}$ and LAFOV scanners, respectively). The results are fitted using a second order polynomial (CIEEE; reproduced with permission [1]).

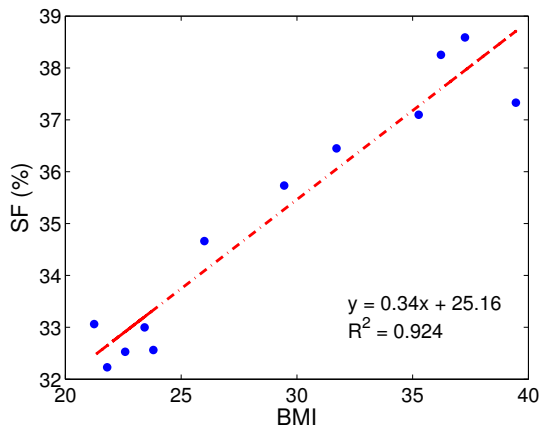

Figure 6: Plot of the scatter fraction obtained from simulations of the anthropomorphic phantom in the LAFOV PET scanner as a function of phantom BMI. 


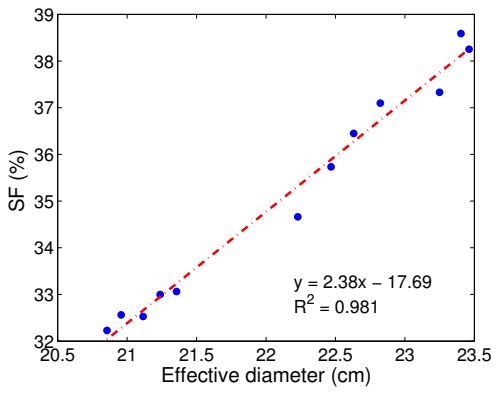

(a) Anthropomorphic SF vs. ED

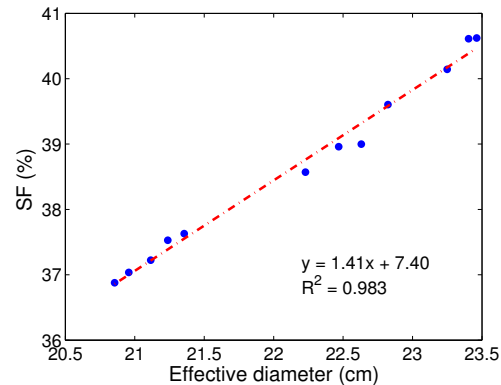

(b) Cylindrical SF vs. ED

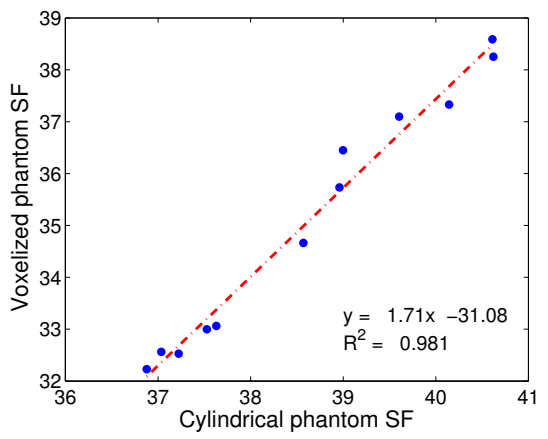

(c) Anthropomorphic vs. cylindrical SF

Figure 7: Plots of scatter fractions obtained with voxelised anthropomorphic scatter phantoms and corresponding cylindrical phantoms, expressed as a function of effective diameter (C)IEEE; reproduced with permission [1]). The correlation between the scatter fractions obtained for both phantom types is also shown.

Table 4: Scatter fractions obtained from uniformly-filled cylindrical phantoms with diameters equivalent to the effective diameters of a set of anthropomorphic phantoms with different BMIs using the LAFOV PET scanner.

\begin{tabular}{lll}
\hline Phantom number $\left(\mathrm{BMI}\left(\mathrm{kg} / \mathrm{m}^{2}\right)\right)$ & Cylindrical phantom SF (\%) & Anthropomorphic phantom SF (\%) \\
\hline (a) $(21.8)$ & 36.88 & 32.23 \\
(b) $(23.8)$ & 37.04 & 32.56 \\
(c) $(22.6)$ & 37.22 & 32.53 \\
(d) $(23.4)$ & 37.52 & 32.99 \\
(e) $(21.2)$ & 37.63 & 33.06 \\
(f) $(26.0)$ & 38.57 & 34.66 \\
(g) $(29.4)$ & 38.96 & 35.73 \\
(h) $(31.7)$ & 38.99 & 36.4 \\
(i) $(35.3)$ & 39.60 & 37.01 \\
(j) $(39.5)$ & 40.14 & 37.33 \\
(k) $(37.3)$ & 40.61 & 38.59 \\
(l) $(36.2)$ & 40.62 & 38.25 \\
\hline
\end{tabular}




\section{Discussion}

The NEMA NU 2-2007 protocol describes a set of standard procedures for evaluating the SF in typical short axial FOV multibed PET scanners. However, none of the phantoms currently defined in either the 2007 version of the standard or in the more recent revisions (published in 2012 and 2018) specifically address scatter in total-body LAFOV PET systems. A Monte Carlo simulation model for a Siemens Biograph $\mathrm{mCT}$ and a LAFOV PET scanner configuration based on the EXPLORER project were developed. The standard NEMA scatter phantom and a NEMA-like scatter phantom extended to cover the full axial FOV of the LAFOV scanner were simulated in each of these scanners, and the results compared with the ground truth.

Our findings demonstrate that the NEMA NU-2-2007 SF protocol, with the line source located at a radial offset of $45 \mathrm{~mm}$, provides an overestimate of the SF compared to the ground truth scatter obtained from a uniformly-filled cylindrical phantom. Furthermore, this overestimate not only occurs for extreme total-body LAFOV scanners, but also to shorter-bore whole body PET systems such as the Siemens Biograph mCT scanner as previously established by Ferrero on a BGO system [42].

The NEMA phantom has not been designed with total-body PET in mind, and does not accurately reflect the situation in which a patient will extend along the entire length of the scanner bore. It was found that a better estimate of the ground truth SF is provided for both scanners when the radial offset is increased to approximately $60 \%$ or $80 \%$ of the phantom radius (depending on whether the ground truth was taken as the average of the SF reported for all radial offsets, or the SF of a uniformly-filled phantom of the same size, respectively). This finding is broadly in agreement with results previously reported by Ferrero et al. for the GE Discovery STE scanner [42].

For both scanners, the estimated SF slightly increases and then progressively decreases as a function of increasing radial offset of the line source within the phantom. The SF is higher for the LAFOV PET compared to the mCT PET scanner. The SFs were estimated using direct binning from the available scatter and prompt counts, instead of following the NEMA NU-2 2007 procedure, since the difference between the SF estimation determined by the NEMA procedure and by direct binning of the Monte Carlo data are within 1\% when using a sufficient number of true coincidence events as reported by Schmidtlein et al. [53].

A strong positive correlation was found between the SFs obtained with the anthropomorphic phantoms and equivalent-volume cylindrical phantoms for LAFOV PET scanners. A slightly weaker correlation between the BMIs of the anthropomorphic phantoms and the SF was also observed. The accuracy of the cylindrical model was best for the highest BMI ranges (with an overestimated SF of around $+2-2.5 \%$ ) and worst for the lower BMIs (an overestimation of $+4.5-4.7 \%)$. The CDC reports that in 2015-2016, the average adult $(>20$ years of age) male in the United States has a height of $1.75 \mathrm{~m}$ and a weight of $89.5 \mathrm{~kg}$, corresponding to a BMI of $29.1 \mathrm{~kg} / \mathrm{m}^{2}$, while the average adult US 
woman has a height of $1.69 \mathrm{~m}$ and a weight of $77.4 \mathrm{~kg}$, corresponding to a BMI of $29.6 \mathrm{~kg} / \mathrm{m}^{2}$ [54]. Considering BMI alone, this most closely corresponds to phantom ( $\mathrm{g}$ ) in both cases (effective diameter $224.7 \mathrm{~mm}$ ). This is only a good match in terms of height for the average female; the average male is approximately $13 \mathrm{~cm}$ taller than the average female. Our results suggest that these will provide an estimate of the expected scatter fraction with an error of $3.2 \%$. It is important to note that scatter is also strongly dependent on the specific radiotracer uptake, which will impact the actual scatter fraction which occurs in each patient - BMI alone is strongly positively correlated with scatter fraction but not the sole determinant, and for practical reasons a cylinder with a narrower effective diameter (and lower BMI) than the equivalent average adult male or female may be preferred. The use of phantom geometries with more realistic non-uniform ${ }^{18} \mathrm{~F}$ distributions for scatter fraction estimation remains an open area for investigation.

\section{Conclusions}

This investigation determined that the SF obtained using the NEMA NU 2-2007 protocol overestimated the actual SF (in comparison to a cylindrical phantom of uniform activity) when the line source is positioned at the recommended radial offset of $45 \mathrm{~mm}$. The study to determine the optimal radial displacement for line source in a solid PET phantom for accurate SF measurements in both the mCT and LAFOV PET scanners showed that the offset which precisely corresponds to the SF obtained with a uniform phantom of the same size is approximately $80 \%$ of the phantom radius for both scanners - the same as reported for the GE Discovery STE scanner [42].

Alternatively, the radial offset which yielded a SF corresponding to the average of all radial displacements from the centre to the edge of the scatter phantom was approximately $60 \%$ of the phantom radius. The SF increases as the object size increases; high correlation is observed between the SF obtained with anthropomorphic phantoms and an equivalent-volume cylindrical phantom for the LAFOV PET scanner.

An additional simulation study was performed to estimate the scatter fraction for the long axial field-of-view PET scanner by simulating different cylindrical and anthropomorphic digital phantoms to characterise the scatter fraction within the total body imaging scanner. Twelve cylindrical phantoms were simulated, each with length of $200 \mathrm{~cm}$, and diameters equal to the effective diameter for each of the twelve anthropomorphic phantoms containing organs filled with realistic ${ }^{18} \mathrm{~F}$-FDG activity concentrations. The SF increases as the object size increase; a strong correlation is observed between the scatter fraction obtained with anthropomorphic phantoms and an equivalent-volume cylindrical phantom for the LAFOV PET scanner. A BMI range between 18.5 and 25 is regarded as healthy; however, patient BMIs have been steadily increasing over time. The increased scatter fraction which will occur due to increasing patient BMI is a point which should be considered in future revisions of scatter fraction esti- 
mation protocols, if they are to reliably estimate scatter fraction with realistic patient dimensions.

\section{Acknowledgements}

This work was supported in part by the Swiss National Science Foundation under grant SNFN 320030_176052 and the Private Foundation of Geneva University Hospitals under Grant RC-06-01, by the Australian Government Research Training Program Scholarship and the ARCLab facility at UTS.

\section{References}

[1] Ghabrial, A., Franklin, D., and Zaidi, H. (November, 2018) Characterization of the scatter component in large axial field-of-view PET scanners: a Monte Carlo simulation study. In 2018 IEEE Nuclear Science Symposium and Medical Imaging Conference Proceedings (NSS/MIC) IEEE.

[2] Cherry, S. R., Jones, T., Karp, J. S., Qi, J., Moses, W. W., and Badawi, R. D. (September, 2017) Total-Body PET: Maximizing Sensitivity to Create New Opportunities for Clinical Research and Patient Care. J Nucl Med, 59(1), 3-12.

[3] Badawi, R. D., Shi, H., Hu, P., Chen, S., Xu, T., Price, P. M., Ding, Y., Spencer, B. A., Nardo, L., Liu, W., Bao, J., Jones, T., Li, H., and Cherry, S. R. (February, 2019) First Human Imaging Studies with the EXPLORER Total-Body PET Scanner. J Nucl Med, 60(3), 299-303.

[4] Badawi, R., Kohlmyer, S., Harrison, R., Vannoy, S., and Lewellen, T. (June, 2000) The effect of camera geometry on singles flux, scatter fraction and trues and randoms sensitivity for cylindrical 3D PET-a simulation study. IEEE T Nucl Sci, 47(3), 1228-1232.

[5] Watanabe, M., Shimizu, K., Omura, T., Sato, N., Takahashi, M., Kosugi, T., Ote, K., Katabe, A., Yamada, R., Yamashita, T., et al. (2003) A highthroughput whole-body PET scanner using flat panel PS-PMTs. In Nuclear Science Symposium Conference Record, 2003 IEEE IEEE Vol. 4, pp. 24422446.

[6] Conti, M., Bendriem, B., Casey, M., Eriksson, L., Jakoby, B., et al. (2006) Performance of a high sensitivity PET scanner based on LSO panel detectors. IEEE T Nucl Sci, 53(3), 1136-1142.

[7] Crespo, P., Reis, J., Couceiro, M., Blanco, A., Ferreira, N. C., Marques, R. F., Martins, P., and Fonte, P. (June, 2012) Whole-Body Single-Bed Time-of-Flight RPC-PET: Simulation of Axial and Planar Sensitivities With NEMA and Anthropomorphic Phantoms. IEEE T Nucl Sci, 59(3), 520-529. 
[8] Poon, J. K., Dahlbom, M. L., Moses, W. W., Balakrishnan, K., Wang, W., Cherry, S. R., and Badawi, R. D. (June, 2012) Optimal whole-body PET scanner configurations for different volumes of LSO scintillator: a simulation study. Phys Med Biol, 57(13), 4077-4094.

[9] Berg, E., Zhang, X., Bec, J., Judenhofer, M. S., Patel, B., Peng, Q., Kapusta, M., Schmand, M., Casey, M. E., Tarantal, A. F., Qi, J., Badawi, R. D., and Cherry, S. R. (February, 2018) Development and Evaluation of mini-EXPLORER: A Long Axial Field-of-View PET Scanner for Nonhuman Primate Imaging. J Nucl Med, 59(6), 993-998.

[10] Lv, Y., Lv, X., Liu, W., Judenhofer, M. S., Zwingenberger, A., Wisner, E., Berg, E., McKenney, S., Leung, E., Spencer, B. A., Cherry, S. R., and Badawi, R. D. (March, 2019) Mini EXPLORER II: a prototype highsensitivity PET/CT scanner for companion animal whole body and human brain scanning. Phys Med Biol, 64(7), 075004.

[11] Zhang, X., Zhou, J., Cherry, S. R., Badawi, R. D., and Qi, J. (February, 2017) Quantitative image reconstruction for total-body PET imaging using the 2-meter long EXPLORER scanner. Phys Med Biol, 62(6), 2465-2485.

[12] Mikhaylova, E., Tabacchini, V., Borghi, G., Mollet, P., D’Hoe, E., Schaart, D. R., and Vandenberghe, S. (October, 2017) Optimization of an ultralowdose high-resolution pediatric PET scanner design based on monolithic scintillators with dual-sided digital SiPM readout: a simulation study. Phys Med Biol, 62(21), 8402-8418.

[13] Viswanath, V., Daube-Witherspoon, M., Schmall, J., Surti, S., Werner, M., Muehllehner, G., Geagan, M., Perkins, A., and Karp, J. (2017) Development of PET for Total-body Imaging. Acta Phys Pol B, 48(10), 1555.

[14] Bettinardi, V., Presotto, L., Rapisarda, E., Picchio, M., Gianolli, L., and Gilardi, M. C. (September, 2011) Physical Performance of the new hybrid PET/CT Discovery-690. Med Phys, 38(10), 5394-5411.

[15] Michopoulou, S., O'Shaughnessy, E., Thomson, K., and Guy, M. J. (March, 2019) Discovery Molecular Imaging Digital Ready PET/CT performance evaluation according to the NEMA NU2-2012 standard. Nucl Med Commun, 40(3), 270-277.

[16] Gopal B. Saha, P. (2016) Basics of PET Imaging, Springer International Publishing, .

[17] Surti, S., Kuhn, A., Werner, M. E., Perkins, A. E., Kolthammer, J., and Karp, J. S. (2007) Performance of Philips Gemini TF PET/CT scanner with special consideration for its time-of-flight imaging capabilities. $J \mathrm{Nucl}$ Med, 48(3), 471-480. 
[18] Rausch, I., Ruiz, A., Valverde-Pascual, I., Cal-González, J., Beyer, T., and Carrio, I. (October, 2018) Performance Evaluation of the Vereos PET/CT System According to the NEMA NU2-2012 Standard. J Nucl Med, 60(4), $561-567$.

[19] Kaneta, T., Ogawa, M., Motomura, N., Iizuka, H., Arisawa, T., HinoShishikura, A., Yoshida, K., and Inoue, T. (October, 2017) Initial evaluation of the Celesteion large-bore PET/CT scanner in accordance with the NEMA NU2-2012 standard and the Japanese guideline for oncology FDG $\mathrm{PET} / \mathrm{CT}$ data acquisition protocol version 2.0. EJNMMI Res, 7(1).

[20] Gonias, P., Bertsekas, N., Karakatsanis, N., Saatsakis, G., Gaitanis, A., Nikolopoulos, D., Loudos, G., Papaspyrou, L., Sakellios, N., Tsantilas, X., Daskalakis, A., Liaparinos, P., Nikita, K., Louizi, A., Cavouras, D., Kandarakis, I., and Panayiotakis, G. (February, 2007) Validation of a GATE model for the simulation of the Siemens biograph ${ }^{\text {TM }} 6$ PET scanner. Nucl. Instrum. Methods. Phys. Res. A, 571(1-2), 263-266.

[21] Jakoby, B., Bercier, Y., Conti, M., Casey, M., et al. (2011) Physical and clinical performance of the mCT time-of-flight PET/CT scanner. Phys Med Biol, 56(8), 2375.

[22] van Sluis, J., de Jong, J., Schaar, J., Noordzij, W., van Snick, P., Dierckx, R., Borra, R., Willemsen, A., and Boellaard, R. (January, 2019) Performance Characteristics of the Digital Biograph Vision PET/CT System. $J$ Nucl Med, 60(7), 1031-1036.

[23] Spencer, B., Schmall, J., Berg, E., Deng, Z., Tang, S., Dong, Y., Leung, E., Lv, Y., Liu, W., Bao, J., Li, H., Jones, T., Badawi, R., and Cherry, S. Performance evaluation of the EXPLORER total-body PET/CT scanner based on NEMA NU-2 2018 standard with additional tests for extended geometry. IEEE Nuclear Science Symposium and Medical Imaging Conference (NSS/MIC) (October, 2019).

[24] Komarov, S. A., Wu, H., Keesing, D. B., O'Sullivan, J. A., and Tai, Y.-C. (June, 2010) Compton Scattering in Clinical PET/CT With High Resolution Half Ring PET Insert Device. IEEE T Nucl Sci, 57(3), 1045-1051.

[25] Zaidi, H. and Montandon, M.-L. (2007) Scatter compensation techniques in PET. PET clinics, 2(2), 219-234.

[26] Teuho, J., Saunavaara, V., Tolvanen, T., Tuokkola, T., Karlsson, A., Tuisku, J., and Teräs, M. (March, 2017) Quantitative Evaluation of 2 Scatter-Correction Techniques for18F-FDG Brain PET/MRI in Regard to MR-Based Attenuation Correction. J Nucl Med, 58(10), 1691-1698.

[27] Berker, Y., Maier, J., and Kachelries, M. (November, 2018) Deep Scatter Estimation in PET: Fast Scatter Correction Using a Convolutional Neural Network. In 2018 IEEE Nuclear Science Symposium and Medical Imaging Conference Proceedings (NSS/MIC) IEEE. 
[28] Werner, M. E., Surti, S., and Karp, J. S. (2006) Implementation and Evaluation of a 3D PET Single Scatter Simulation with TOF Modeling. In 2006 IEEE Nuclear Science Symposium Conference Record IEEE.

[29] Watson, C. (October, 2007) Extension of Single Scatter Simulation to Scatter Correction of Time-of-Flight PET. IEEE T Nucl Sci, 54(5), 1679-1686.

[30] Zaidi, H. (April, 1999) Relevance of accurate Monte Carlo modeling in nuclear medical imaging. Med Phys, 26(4), 574-608.

[31] Castiglioni, I., Cremonesi, O., Gilardi, M., Bettinardi, V., Rizzo, G., Savi, A., Bellotti, E., and Fazio, F. (1999) Scatter correction techniques in 3D PET: a Monte Carlo evaluation. IEEE T Nucl Sci, 46(6), 2053-2058.

[32] Holdsworth, C., Badawi, R., Santos, P., Van den Abbeele, A., Hoffman, E., and El Fakhri, G. (2003) Evaluation of a Monte Carlo scatter correction in clinical 3D PET. In Nuclear Science Symposium Conference Record, 2003 IEEE IEEE Vol. 4, pp. 2540-2544.

[33] Hirano, Y., Koshino, K., and Iida, H. (April, 2017) Influences of 3D PET scanner components on increased scatter evaluated by a Monte Carlo simulation. Phys Med Biol, 62(10), 4017-4030.

[34] Zaidi, H. (2000) Comparative evaluation of scatter correction techniques in 3D positron emission tomography. Eur J Nucl Med Mol, 27(12), 1813-1826.

[35] Zaidi, H. and Koral, K. F. (May, 2004) Scatter modelling and compensation in emission tomography. Eur J Nucl Med Mol, 31(5), 761-782.

[36] Gaens, M., Bert, J., Pietrzyk, U., Shah, N. J., and Visvikis, D. (2013) GPUaccelerated Monte Carlo based scatter correction in brain PET/MR. In Nuclear Science Symposium and Medical Imaging Conference (NSS/MIC), 2013 IEEE IEEE pp. 1-3.

[37] Eriksson, L., Townsend, D., Eriksson, M., Melcher, C., , et al. (2004) Experience with scintillators for PET: towards the fifth generation of $\{\mathrm{PET}\}$ scanners. Nucl Instrum Methods Phys Res A, 525(1-2), 242 - 248.

[38] MacDonald, L. R., Harrison, R. L., Alessio, A. M., Hunter, W. C. J., Lewellen, T. K., and Kinahan, P. E. (May, 2011) Effective count rates for PET scanners with reduced and extended axial field of view. Phys Med Biol, 56(12), 3629-3643.

[39] Badawi, R., Adam, L.-E., and Zimmerman, R. (2001) A simulation-based assessment of the revised NEMA NU-2 $70 \mathrm{~cm}$ long test phantom for PET. In 2001 IEEE Nuclear Science Symposium Conference Record (Cat. No.01CH37310) IEEE.

[40] Jan, S., Benoit, D., Becheva, E., Carlier, T., et al. (2011) GATE V6: a major enhancement of the GATE simulation platform enabling modelling of CT and radiotherapy. Phys Med Biol, 56(4), 881. 
[41] NEMA Standards Publication NU 2-2007: Performance measurements of positron emission tomographs. (2007).

[42] Ferrero, A., Poon, J. K., Chaudhari, A. J., MacDonald, L. R., and Badawi, R. D. (2011) Effect of object size on scatter fraction estimation methods for PET - a computer simulation study. IEEE T Nucl Sci, 58(1), 82-86.

[43] Poon, J. K., Dahlbom, M. L., Casey, M. E., Qi, J., Cherry, S. R., and Badawi, R. D. (2015) Validation of the SimSET simulation package for modeling the Siemens Biograph mCT PET scanner. Phys Med Biol, 60(3), N35.

[44] Ghabrial, A., Franklin, D., and Zaidi, H. (June, 2018) A Monte Carlo simulation study of the impact of novel scintillation crystals on performance characteristics of PET scanners. Phys Medica, 50, 37-45.

[45] NEMA Standards Publication NU 2-2012: Performance measurements of positron emission tomographs. (2012).

[46] NEMA Standards Publication NU 2-2018: Performance measurements of positron emission tomographs. (2018).

[47] Spencer, B. A., Berg, E., Schmall, J. P., Omidvari, N., Leung, E. K., Abdelhafez, Y. G., Tang, S., Deng, Z., Dong, Y., Lv, Y., Bao, J., Liu, W., Li, H., Jones, T., Badawi, R. D., and Cherry, S. R. (October, 2020) Performance evaluation of the uEXPLORER Total-body PET/CT scanner based on NEMA NU 2-2018 with additional tests to characterize long axial field-of-view PET scanners. J Nucl Med, p. jnumed.120.250597.

[48] Thielemans, K., Tsoumpas, C., Mustafovic, S., Beisel, T., et al. (2012) STIR: software for tomographic image reconstruction release 2. Phys Med Biol, $\mathbf{5 7}(4), 867$.

[49] Akhavanallaf, A., Xie, T., and Zaidi, H. (2019) Development of a library of adult computational phantoms based on anthropometric indexes. IEEE T Rad Plasma Med Sci, 3(1), 65-75.

[50] Valentin, J. Radiation dose to patients from radiopharmaceuticals : a third amendment to ICRP publication 53 pp. 85-86 Published for the International Commission on Radiological Protection by Elsevier Oxford (2009).

[51] Vauclin, S., Michel, C., Buvat, I., Doyeux, K., Edet-Sanson, A., Vera, P., Gardin, I., and Hapdey, S. (2015) Monte-Carlo simulations of clinically realistic respiratory gated 18 F-FDG PET: Application to lesion detectability and volume measurements. Comput Methods Programs Biomed, 118(1), 84-93.

[52] Boone, J., Strauss, K., Cody, D., et al. Size-specific dose estimates (SSDE) in pediatric and adult body CT examinations: AAPM report no. 204. American Association of Physicists in Medicine website. (2013). 
[53] Schmidtlein, C. R., Kirov, A. S., Nehmeh, S. A., Erdi, Y. E., et al. (2006) Validation of GATE Monte Carlo simulations of the GE Advance/Discovery LS PET scanners. Med Phys, 33(1), 198-208.

[54] Fryar, C., Kruszon-Moran, D., Gu, Q., and Ogden, C. (December, 2018) Mean Body Weight, Height, Waist Circumference, and Body Mass Index Among Adults: United States, 1999-2000 Through 2015-2016. Natl Health Stat Report, 2018(122), 1-16. 